\title{
Acoustic Noise Prediction and Reduction Reasearch of Helicopter Ducted Tail Rotor
}

\author{
Chaopu Zhang ${ }^{a}$, Mingxu Yi ${ }^{b}$, Jun Huang ${ }^{c}$ and Liu Yang ${ }^{d}$ \\ School of Aeronautic Science and Engineering, Beihang University, Beijing 100191, China \\ azhangchaopu@buaa.edu.cn, byimingxu88@163.com, cjunhuang1964@163.com, \\ doisy66@163.com
}

\begin{abstract}
This research aims to study the acoustic noise prediction and reduction of helicopter ducted tail rotor. A thin-body BEM/FW-H method is developed to accurately predict the noise in this paper. It is a hybrid method combining computational aero acoustics with Boundary Element Method (BEM) and can be used efficiently to predict the propagation of sound wave in the duct. And two main geometry parameters are modified to investigate the noise reduction of ducted tail rotor by using the numerical prediction method. The acoustic performance of different modification tasks is discussed. These results verify the efficiency of design parameters modification to reduce acoustic noise of ducted tail rotor.
\end{abstract}

Keywords: Acoustic noise; Noise reduction; ducted tail rotor.

\section{Introduction}

Ducted tail rotor is a device of great importance to a single main rotor helicopter. The noise of ducted tail rotor is an aeroacoustic problem, which is concerned with the generation and propagation of acoustic noise. As for acoustic noise of blade and duct, previous researches on the generation and propagation of the noise of rotor and axial fan have yielded large amount of good results. Generally, the noise source is expressed using acoustic analogy and the flow field is solved using Computational Fluid Dynamics (CFD) or measured by experimental means [1,2]. Over the last two decades, the Boundary Element Method (BEM) has become a valuable modeling especially for problems in acoustics and noise control.Improved understanding of the acoustic noise reduction can lead to quieter helicopter designs. Some researchers have studied the design parameters improvement for acoustic noise reduction of helicopter. Hu et al. [3] studied blade-sweep blade-vortex interaction noise reduction design and found that a switch of noise level increase on the retreating side to the reduction in maximum noise level on the advancing side greatly favored noise reduction on the whole, with the 'hot' noise spots optimized to less 'hot' noise region. Chae et al. [4]made efforts to improve the acoustic performance of helicopter rotor based on shape parameter optimization and found variations in parameters including the taper ratios, the swept back, the tip chord length, the protrusion shape, the camber, and the thickness of the root airfoil would bring significant change of rotor aeroacoustic performance. Wang et al. [5] analyzed the acoustic noise of rotor with unconventional blade tips and demonstrate the unconventional CLOR-II blade tip can reduce the HSI noise of a rotor significantly.

This paper focuses on a discussion of ducted tail rotor modification based on noise reduction. The hybrid method combining thin-body BEM and FW-H method is developed to predict the acoustic noise of ducted tail rotor in this research. Then two main geometry parameters are modified to reduce the noise of ducted tail rotor. The acoustic performance of different modification tasks is discussed and shown in this research.

\section{Numerical methods}

The noise of ducted tail rotor is generated from the pressure fluctuation of the blade surface, which is caused by the unsteady aerodynamic forces. The noise of ducted tail rotor is composed of incident sound generated by blade and scattered sound generated by duct. The thin-body BEM/FW-H numerical method is divided into three steps: firstly, the sound source generated from the pressure 
fluctuations of the blade surface is calculated using CFD method; secondly, the free-field sound pressure is calculated using FW-H method, and then it's transformed into sound pressure in frequency domain using Fast Fourier Transform (FFT) method; lastly, the scattering effect of the duct wall is solved using thin-body BEM method, and the radiation and propagation of the sound source is calculated.

\subsection{Unsteady flow calculation.}

Accurate calculation of the flow field is very essential for the calculation of acoustic noise. Due to the constant rotary speed, we focus on the flow field in stable state. Therefore, the unsteady Navier-Stokes equation is adopted here to calculate the flow field [6].

In this research, the flow field properties are calculated by using the commercial CFD code FLUENT 14.0, which has a lot of turbulence models such as model, SST model and LES model. The standard model is used in this research for its reasonable solving accuracy and fewer resources compared with SST model and LES model.

The no-slip wall is adopted in this aerodynamic simulation. The SIMPLEC discretization schemes are used to discretize the equation of pressure-velocity coupling. The rotating speed of ducted tail rotor modification is $2310 \mathrm{r} / \mathrm{min}$. And the time step in relation to the rotating speed is given in order to obtain enough calculated results per blade passing time.

\subsection{Free field acoustic source.}

Acoustic analogy can be used to study the problem of aerodynamically generated noise, and the FW-H equation is adopted to calculate the free-field acoustic noise [7, 8]. FW-H equation can be solved by using the formulation of Farassat 1A, which is given as follows:

$$
p^{\prime}(x, t)=p_{T}{ }^{\prime}(x, t)+p_{L}{ }^{\prime}(x, t)
$$

With

$$
\begin{aligned}
4 \pi p_{T}{ }^{\prime}(x, t) & =\int_{S}\left[\frac{\rho_{0}\left(\dot{U}_{n}+U_{\dot{n}}\right)}{r\left(1-M_{r}\right)^{2}}\right]_{r e t} d S+\int_{S}\left[\frac{\rho_{0} U_{n}\left(r_{i} \dot{M}_{i}+c_{0} M_{r}-c_{0} M^{2}\right)}{r^{2}\left(1-M_{r}\right)^{3}}\right]_{r e t} d S \\
4 \pi p_{L}{ }^{\prime}(x, t) & =\frac{1}{c_{0}} \int_{S}\left[\frac{\dot{L}_{r}}{r\left(1-M_{r}\right)^{2}}\right]_{r e t} d S+\int_{S}\left[\frac{L_{r}-L_{M}}{r^{2}\left(1-M_{r}\right)^{2}}\right]_{r e t} d S \\
& +\frac{1}{c_{0}} \int_{S}\left[\frac{L_{r}\left(r \dot{M}_{r}+c_{0} M_{r}-c_{0} M^{2}\right)}{r^{2}\left(1-M_{r}\right)^{3}}\right]_{r e t} d S
\end{aligned}
$$

Where $p_{T}{ }^{\prime}(x, t)$ represents thickness sound pressure and $p_{L}{ }^{\prime}(x, t)$ represents loading sound pressure. $\rho_{0}$ And $c_{0}$ are the fluid and sound speed, respectively. $L$ Represents the aerodynamic pressure with $L_{i}=P_{i j} \hat{n}_{j}+\rho u_{i}\left(u_{n}-v_{n}\right) \cdot u_{i}$ Is the fluid velocity and $v_{i}$ represents the velocity of the surface. The relative speed $\left(u_{i}-v_{i}\right)=0$ and $U_{i}$ is reduced to $U_{i}=v_{i}$ when the control surface is solid. $\boldsymbol{M}$ Represents local Mach number vector of source with respect to a frame fixed to the undisturbed medium, with components $M_{i}$. And the subscript $r$ denotes projection onto the source observer direction. The subscript $M$ denotes projection on the Mach number vector.

After the free-field sound pressure is calculated using FW-H method, it is transformed into sound pressure in frequency domain by FFT method.

\subsection{Acoustic thin-body BEM for sound propagation.}

The BEM method is used to calculate the far-field sound pressure. Due to the scattering effect of the duct wall, the total sound pressure is the superposition of incident pressure and scattered pressure $[9,10]$. The incident pressure in frequency domain can be solved using the method mentioned in previous sections, and the scattered pressure can be solved using BEM.

$$
p_{\text {sum }}^{\prime}=p_{i}^{\prime}+p_{s}^{\prime}
$$


Where $p_{i}^{\prime}$ and $p^{\prime}{ }_{s}$ separately denote incident sound pressure and scattered sound pressure in the frequency domain. In this research, the scattered sound pressure is calculated using the thin-body boundary integral equation. The calculated domain is shown in Fig. 1, the surface of the duct wall is expressed by $S$. Since the duct is not close, an imaginary surface $S_{i}$ is constructed to divide the acoustic domain into an interior domain $V^{-}$and an exterior domain $V^{+}$. The sound pressure on the inside of the closed surface is denoted as $p^{-}$while outside is $p^{+}$. The integral equation is

$$
\begin{aligned}
& \int_{S}\left(p^{+}-p^{-}\right) \frac{\partial \psi}{\partial n} d S=\frac{1}{2}\left[p^{+}(x)-p^{-}(x)\right] \quad x \in S \\
& \int_{S}\left(p^{+}-p^{-}\right) \frac{\partial \psi}{\partial n} d S=p(x) \quad x \in V^{-} \cup V^{+}
\end{aligned}
$$

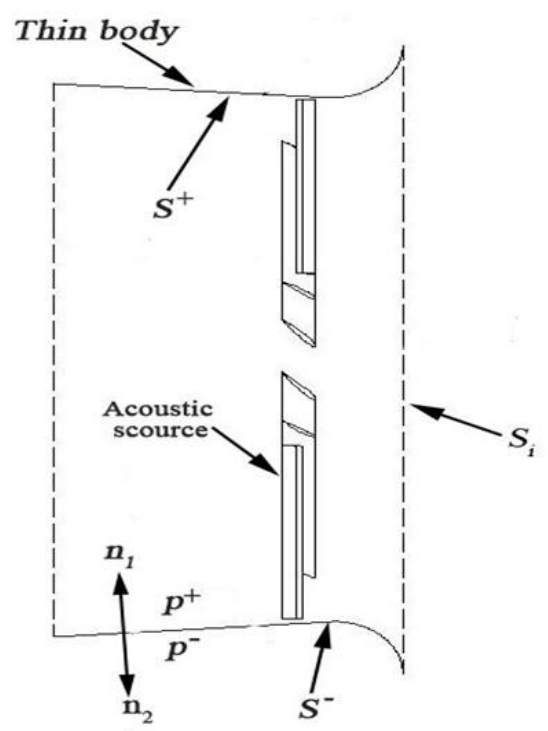

Fig. 1 The schematic diagram of acoustic scattering by a thin body

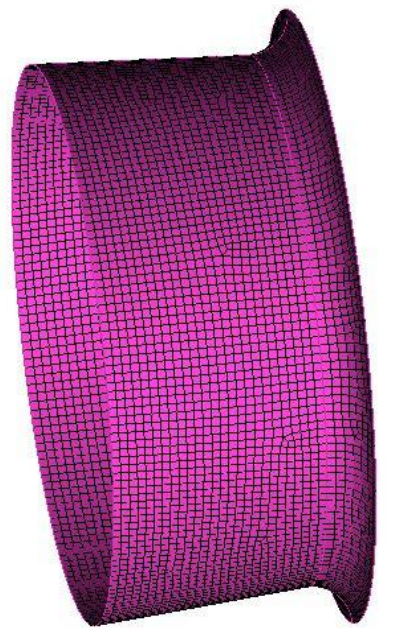

Fig. 2 Mesh of duct used in BEM

In order to calculate the scattered sound pressure, the thin-body neutral surface $S$ is discretized into a number of elements. Using the Linear BEM, the sound pressure jump $\left(p^{+}-p^{-}\right)$can be calculated by solving a set of equations. Then the scattered sound pressure at any elements can be solved directly by Eq. (5) and Eq. (6). The mesh of duct surface used in BEM is shown as Fig. 2.

An effort to realize the numerical noise prediction of ducted tail rotor was made through solving Eq. (4). 


\section{Model establishment}

\subsection{Geometric configuration and grid generation.}

A ducted tail rotor system is designed and tested to analyze and reduce its acoustic noise in this part. The virtual prototype model of ducted tail rotor is set up in Tab. 1, and its geometric model is shown in Fig. 3.

Table 1 Parameters of benchmark model of ducted tail rotor

\begin{tabular}{cc}
\hline Structural parameter & Size \\
\hline Rotor radius R & $750 \mathrm{~mm}$ \\
Rotor blade chord $C$ & $110 \mathrm{~mm}$ \\
Rotor blade airfoil & NACA23012 \\
Blade angle $\phi$ & $40^{\circ}$ \\
Number of rotor blades $B$ & 11 \\
Rotor hub diameter(0.7R) & $525 \mathrm{~mm}$ \\
Duct spread angle $\theta_{\text {dif }}$ & $5^{\circ}$ \\
Duct lip radius Rlip(0.18R) & $135 \mathrm{~mm}$ \\
Duct gap Lgap $(0.015 \mathrm{R})$ & $11.25 \mathrm{~mm}$ \\
Rotor disc position $(0.75 \mathrm{R})$ & $562.5 \mathrm{~mm}$ \\
Length of exhaust section(0.7R) & $525 \mathrm{~mm}$ \\
\hline
\end{tabular}

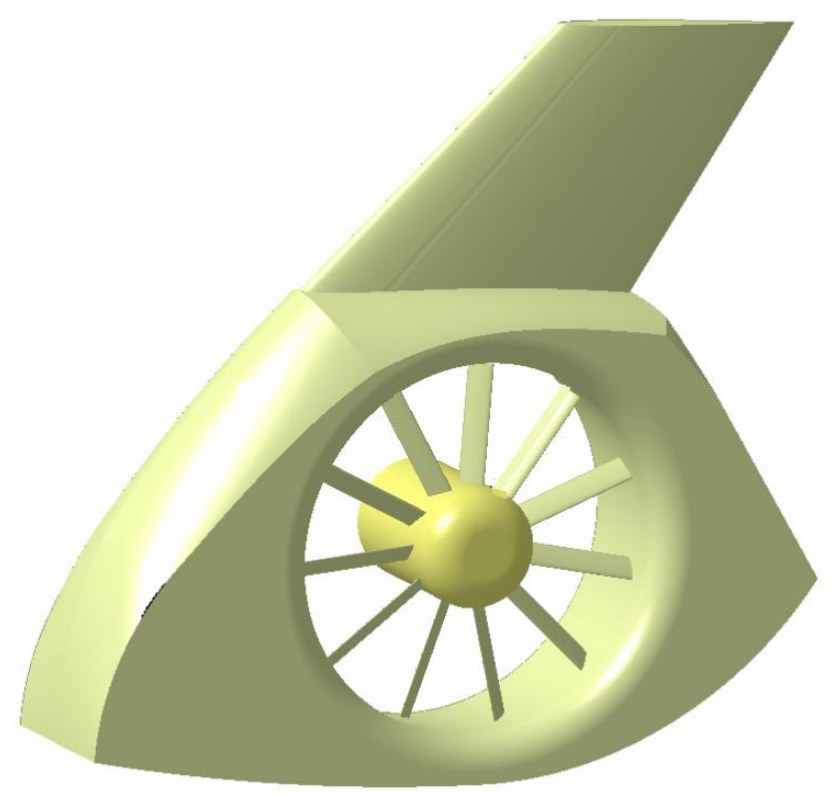

Fig. 3 Geometric model of ducted tail rotor

In the aerodynamics simulation of ducted tail rotor, the whole computation domain is divided into two subdomains: an independent rotational domain for the rotor disk and an outer stationary domain. The stationary domain includes the part of duct and rotor hub. After the geometry of ducted tail rotor is established, the calculation model is meshed by the unstructured triangle mesh, and the grid density can be controlled to improve computational precision. The grids in the rotational domain and duct domain are dense, as shown in Fig. 4. In consideration of the inlet and outlet zone (shown in Fig. 5), the total number of cells in the whole flow field is about 4.2 million. 


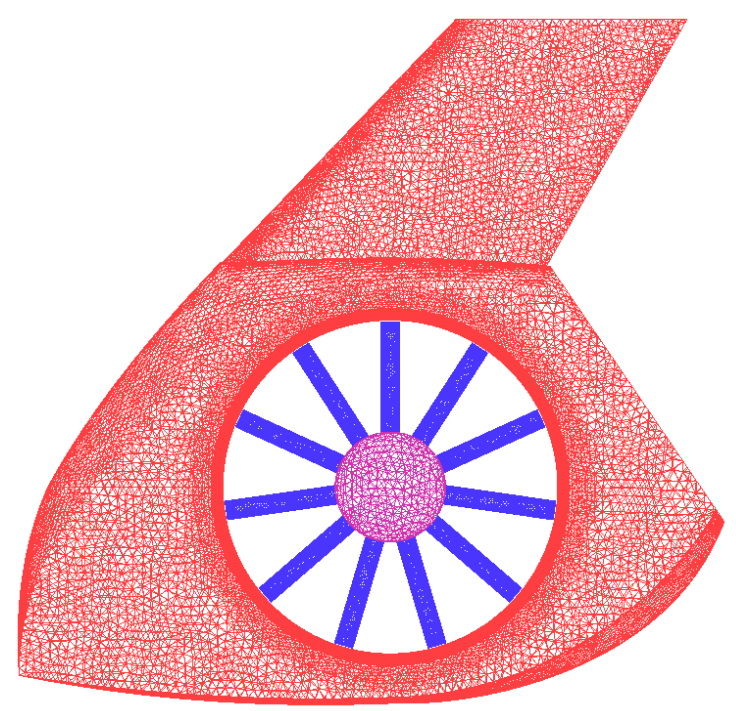

Fig. 4 Gird of ducted tail rotor

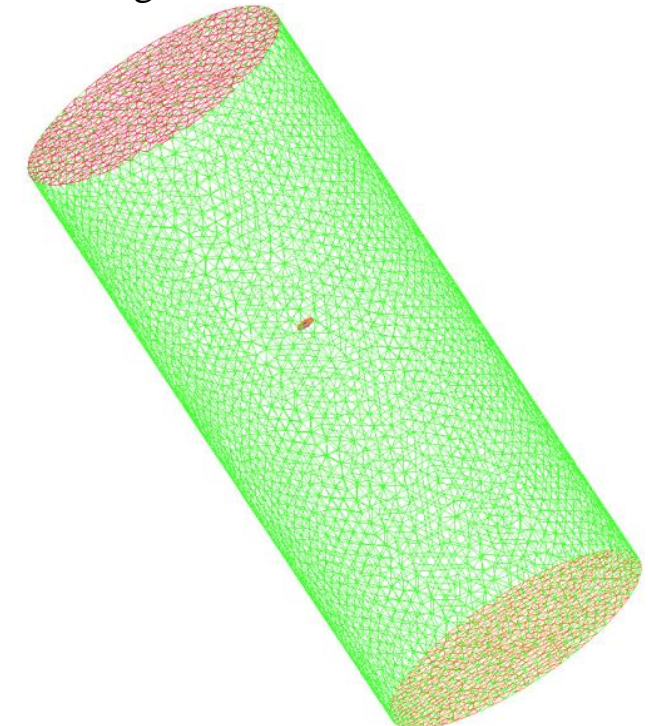

Fig. 5 Gird of the far-field

\subsection{Acoustic noise of ducted tail rotor.}

In this simulation, all of the acoustic noise and SPLs of ducted tail rotor prototype model and the following different modifications are calculated at rotary speed of $2310 \mathrm{rpm}$ at the observation distance of $5 \mathrm{~m}$.

The sound pressure is expressed in decibels (dB), and SPL is calculated by the following equation:

$$
S P L=P_{e} / P_{\text {ref }}
$$

Where $P_{e}$ denotes the predicted pressure, $P_{\text {ref }}$ denotes the reference pressure and equals to $2 \times 10^{-5} \mathrm{~Pa}$.

\section{Applications in acoustic noise reduction}

In this part, the numerical prediction method mentioned above is applied to study the acoustic noise reduction of ducted tail rotor. By analyzing the amendable geometrical parameters that have an obvious influence on acoustic noise, two design parameters including airfoil and chord length are altered to analyze the acoustic characteristics of ducted tail rotor.

The influence on acoustic noise of blade airfoil was considered in the first place. Four commonly used helicopter tail rotor airfoils were selected, i.e. NACA23012, ONERA OA209, BOEING-VERTOL VR-12 and ONERA OA213. The airfoil of original model is NACA23012.Then, 
the influence on acoustic noise from blade angle which was modified from 40-50 degrees was discussed, as shown in Tab. 3. The SPLs ducted tail rotor calculated for various airfoils and blade angles are shown as Fig.6.

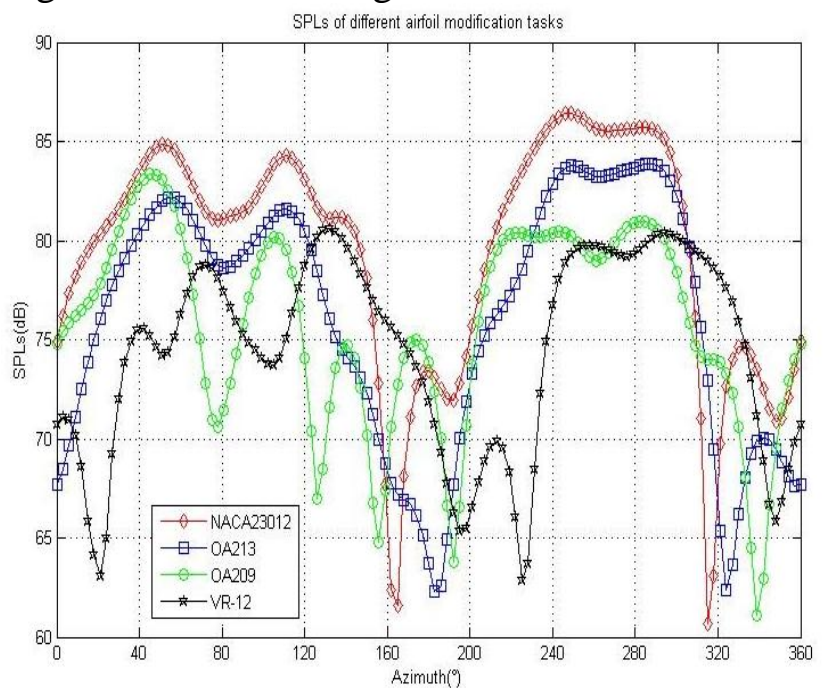

(a)

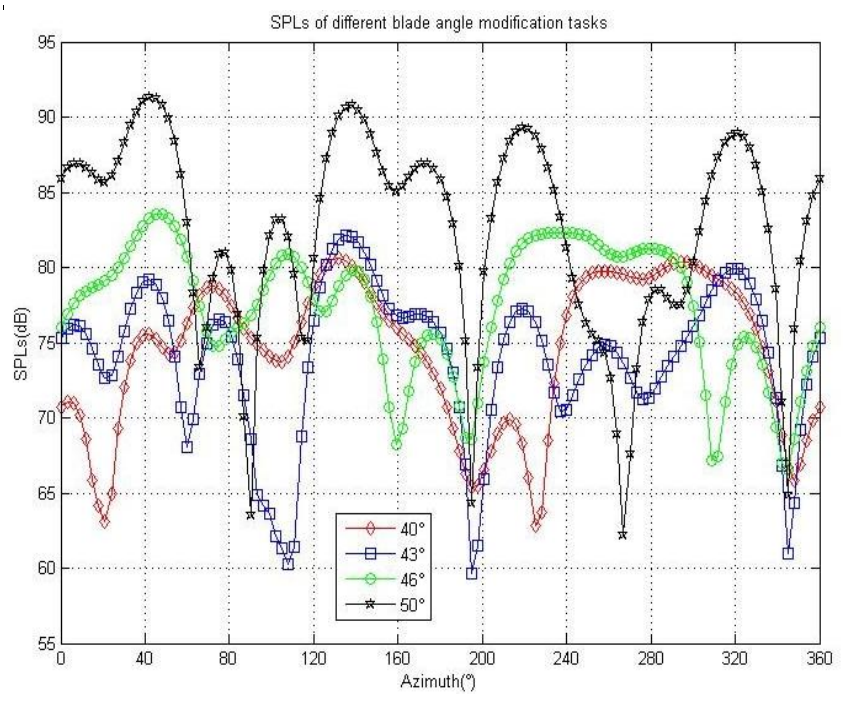

(b)

Fig. 6 SPLs of different airfoil modification tasks (a) and blade angle modification tasks (b)

Table 2 Modification tasks of blade airfoil and corresponding average SPLs

\begin{tabular}{ccccc}
\hline Task & 1 & 2 & 3 & 4 \\
\hline Airfoil & NACA23012 & OA213 & VR-12 & OA209 \\
SPLs (dB) & 79.4 & 76.3 & 74.5 & 75.8 \\
\hline
\end{tabular}

These four airfoils have great similarities in their shape and proximity in their maximum camber. So they have similar thrust. However the SPLs of NACA23012 and ONERA OA213 are slightly higher than ONERA OA209 and BOEING-VERTOL VR-12 (Tab. 2; Fig. 6). On the whole, the lowest noise airfoil is BOEING-VERTOL VR-12, whose SPL is 74.5dB (Tab. 2; Fig. 6).

Table 3 Modification tasks of blade angle and corresponding average SPLs

\begin{tabular}{ccccc}
\hline Task & 1 & 2 & 3 & 4 \\
\hline Blade angle $\left(^{\circ}\right)$ & 40 & 43 & 46 & 50 \\
SPLs $(\mathrm{dB})$ & 74.5 & 73.9 & 77.4 & 82.5 \\
\hline
\end{tabular}

Relatively high angle of attack generates high noise (Tab. 3). In most azimuth angles, when the blade angle is 43 degrees, its acoustic noise is the lowest among the four cases (Fig.6; Tab. 3), so its overall noise is the lowest, with the corresponding SPL being 73.9dB. Thus, the change of blade angle has a big impact on the aerodynamic and acoustic performance of ducted tail rotor.

\section{Summary}

This paper aims to predict and reduce the acoustic noise of helicopter ducted tail rotor. The acoustic characteristics of ducted tail rotor were investigated by using the thin-body BEM/FW-H method. And then the effects of two main design parameters, including airfoil and blade angle were discussed. These design parameters have obvious influence on the acoustic performance of ducted tail rotor. Some meaningful conclusions are drawn on the noise reduction. By modifying the two main design parameters, the SPL decrease by $5.5 \mathrm{~dB}$ compared with the original model. These results demonstrate the validity of design parameters modification of ducted tail rotor in acoustic noise reduction. In the following research, the noise reduction effect of other design parameters can be investigated. 


\section{References}

[1]. D.K.M. Argüelles, O.J.M. Fernández, M.E. Blanco, et al. Numerical prediction of tonal noise generation in an inlet vaned low-speed axial fan using a hybrid aeroacoustic approach. Journal of Mechanical Engineering Science. Vol. 223 (2009) No. 9, p. 2081-2098.

[2]. Y. Wang, G.H. Xu, Q.J. Zhao. Numerical method for predicting rotor aerodynamic noise based on unstructured-grid CFD technology. Acta Aerodynamica Sinica. Vol. 29 (2011) No. 5, p. 559-566.

[3]. H. Hu, L.A. Jordan, J.D. Baeder. Computational simulation and analysis of double-swept blade in BVI noise reduction. Advances in Engineering Software. Vol. 36 (2005) No. 2, p. 67-76.

[4]. S. Chae, K. Yee, C. Yang, et al. Helicopter rotor shape optimization for the improvement of aeroacoustic performance in hover. Journal of Aircraft. Vol. 47(2010) No. 5, p. 1770-1783.

[5]. B. Wang, Q. Zhao, G. Xu, et al. Numerical analysis on noise of rotor with unconventional blade tips based on CFD/Kirchhoff method. Chinese Journal of Aeronautics. Vol. 26 (2013) No. 3, p. 572-582.

[6]. G. Srinivasan, W. McCroskey. Navier-Stokes calculations of hovering rotor flowfields. Journal of Aircraft. Vol. 25 (1988) No. 10, p. 865-874.

[7]. K.S. Brentner, F. Farassat. Analytical Comparison of the Acoustic Analogy and Kirchhoff Formulation for Moving Surfaces. AIAA Journal. Vol. 36 (1998) No. 8, p. 1379-1386.

[8]. D. Fedala, S. Kouidri, R. Rey. Numerical study of time domain analogy applied to noise prediction from rotating blades. Journal of Sound and Vibration. Vol. 321 (2009) No. 3-5, p. 662-679.

[9]. M. Carley. The Sound Field of a Rotor in a Stationary Duct. Journal of Sound and Vibration. Vol. 259 (2003) No. 5, p. 1067-1079.

[10]. B.B. Hu, H. OuYang, Y.D. Wu, et al. Numerical prediction of the interaction noise radiated from an axial fan. Applied Acoustics. Vol. 74 (2013) No. 4, p. 544-552. 